\title{
Self-Aligned Silicon Ring Resonator Optical Modulator with Focused Ion Beam Error Correction
}

\author{
D. J. Thomson ${ }^{1}$, F. Y. Gardes ${ }^{1}$, D. C. Cox $^{2}$, J-M. Fedeli ${ }^{3}$, G. Z. Mashanovich ${ }^{1}$ and G. T. Reed ${ }^{1}$. \\ ${ }^{1}$ Optoelectronics Research Centre, University of Southampton, Southampton, UK. \\ ${ }^{2}$ Advanced Technology Institute, University of Surrey, Guildford, UK. \\ ${ }^{3}$ CEA, LETI, Minatec Campus, 17 Rue des Martyrs, 38054, Grenoble (France) \\ *Corresponding author: d.thomson@soton.ac.uk
}

\begin{abstract}
Ring resonator based silicon optical modulators have a tremendous potential for forming compact and power efficient optical transmitters. In this paper we demonstrate high speed modulation from a device based upon carrier depletion in a self aligned pn junction. Furthermore the role that focused ion beams can play in the error correction of silicon photonic devices is also demonstrated.
\end{abstract}

(C2012 Optical Society of America

OCIS codes: (130.4110) Modulators; (060.4080) Modulation

\section{INTRODUCTION}

High speed, all silicon optical modulators have attracted significant research interest in recent years. This has coincided with rapid performance enhancements with devices reporting data rates up to $50 \mathrm{Gbit} / \mathrm{s}$ recently [1]. Generally the most successful devices have been based upon the plasma dispersion effect [2-3] and use carrier depletion in a reverse biased diode structure to electrically manipulate the free carrier density which is in interaction with the propagating light. Although the plasma dispersion effect can directly cause intensity modulation due to the relationship between free carrier densities and optical absorption, it is more effective to use the accompanying change in the real part of the refractive index to shift the phase of the light and then convert to intensity modulation using a resonant or interference based structure. Mach-Zehnder interferometers (MZI) are commonly used due to their robustness to fabrication tolerances, wide optical bandwidth and thermal insensitivity. On the other hand the interaction length required usually results in large devices, with a large power consumption unless slow light techniques are employed such as in [4-5]. In contrast, phase modulators incorporated into resonant structures such as ring resonators can be very compact and consume relatively little power [6].

Several ring resonator based modulator devices have been demonstrated recently [7-19]. In [11-14] modulation data is presented up to $\sim 40 \mathrm{Gbit} / \mathrm{s}$ in a ring based device. In [15-16] another technique tolerant to alignment errors based upon interleaved pn junctions is presented. In [17] a heater is incorporated with a high speed ring modulator to allow tuning or stabilisation of the resonant wavelength during operation. In [18] a ring resonator modulator is combined with an MZI to ease the sensitivity of the device to fabrication tolerances. Finally, in [19] a differentially driven disk resonator is demonstrated with a power consumption as low as $2 \mathrm{fj} / \mathrm{bit}$.

Recently we have demonstrated a MZI based device with a phase modulator that features a selfaligned pn junction operating at 50Gbit/s [1]. Here we demonstrate not only that the same self-aligned technology can also be applied to a ring resonator based device, but also that it can be more effective since a greater interaction of the light with the region of depleted carriers occurs. A mask design error required the use of a focussed ion beam (FIB) for error correction of the fabricated device. This work demonstrates the value of such a technique in the prototyping of silicon photonic devices and systems.

\section{DEVICE DESIGN AND FABRICATION}

The devices are formed in silicon-on-insulator (SOI) of overlayer thickness $220 \mathrm{~nm}$ and buried oxide thickness $2 \mu \mathrm{m}$. The waveguides therefore have a height of $220 \mathrm{~nm}$, the width is $400 \mathrm{~nm}$ and the slab height is $100 \mathrm{~nm}$. A $1 \mu \mathrm{m}$ thick silicon dioxide top cladding layer is deposited onto the waveguides. The ring resonator has a radius of $6 \mu \mathrm{m}$ and a ring to waveguide separation of $150 \mathrm{~nm}$ in the coupling region. The waveguides leading to the device have a width of $2 \mathrm{um}$ to minimise device access loss. They then taper down to the device waveguide width of $400 \mathrm{~nm}$ over a length of $350 \mu \mathrm{m}$. Figure 1 shows a cross section of the ring resonator modulator. The rib region of the waveguide and the slab to one side is $p$ type doped to an approximate concentration of $3 \mathrm{e} 17 . \mathrm{cm}^{-3}$. The slab on the other side is $\mathrm{n}$ type doped to $\sim 1.5 \mathrm{e} 18 . \mathrm{cm}^{-3}$. These $\mathrm{p}$ and $\mathrm{n}$ type doped slabs extend out to $\mathrm{p}+$ and $\mathrm{n}+$ type regions $\left(\sim 1 \mathrm{e} 20 . \mathrm{cm}^{-3}\right)$ respectively which in turn contact 
to the device electrodes. The $\mathrm{n}$ type region has a higher doping concentration than the $\mathrm{p}$ type region such that depletion extends mainly into the waveguide under reverse bias conditions. The $\mathrm{n}$ type region is positioned on the outside edge of the ring so that as the mode is forced outwards whilst propagating around the ring it has a greater interaction with the region of depleted carriers resulting in a greater modulation efficiency than in the case of the a straight phase modulator. The $\mathrm{p}+$ and $\mathrm{n}+$ regions are situated $450 \mathrm{~nm}$ and $500 \mathrm{~nm}$ from the waveguide edges respectively.

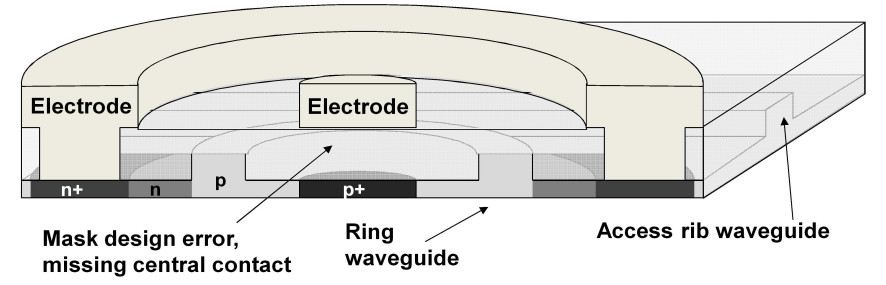

Fig. 1. Self aligned pn junction ring modulator.

Device fabrication was performed in the $200 \mathrm{~mm}$ microelectronics clean room of CEA-LETI. Firstly grating regions are etched into the surface of the wafer to provide a means to couple light to and from the optical waveguides. The active regions and waveguides are then formed. The device is designed to allow for a self- aligned process to be used to align the pn junction with the waveguide [21]. Firstly the active region of the device is implanted with boron. A silicon dioxide layer is then deposited and patterned with the waveguide design. This is firstly used as a hard mask to etch the optical waveguides in the silicon overlayer and then used together with a photoresist window to define the region to be doped n-type. Phosphorus is then implanted into this region. The $\mathrm{p}+$ and $\mathrm{n}+$ regions are then implanted through photoresist windows. A 1um thick silicon dioxide layer is then deposited and contact holes etched down to expose the $\mathrm{p}+$ and $\mathrm{n}+$ regions. Finally, a metal stack of the following materials and thicknesses is deposited and patterned to form the electrodes: $\mathrm{Ti}$ (30nm), TiN (60nm), AlCu (650nm), Ti (10nm) and TiN $(40 \mathrm{~nm})$.

A mask design error meant that the centre contact hole was not defined. As a result the fabricated devices were not electrically active. To rectify this problem a FEI Nova Nanolab 600 focused ion beam (FIB) was used to ion mill a hole down though the centre electrode and upper cladding layer to just above the $\mathrm{p}^{+}$ doped region. The milled hole was stopped just short of the $\mathrm{p}+$ layer, as being very thin $(100 \mathrm{~nm})$ even a few seconds of over exposure from the ion beam would mill beyond it. (a)

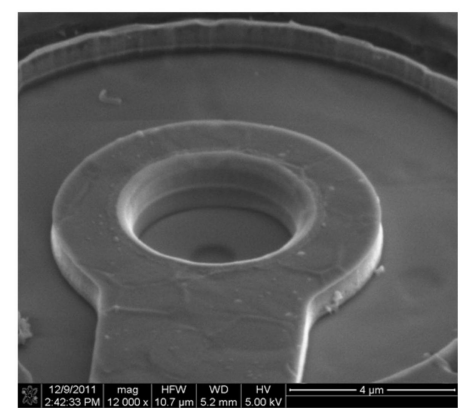

(d)

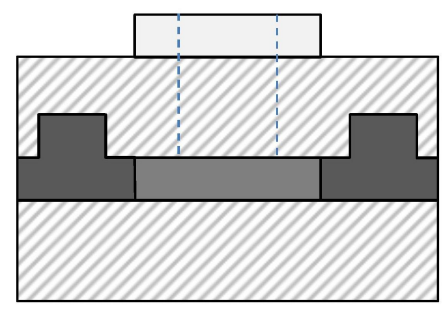

(b)

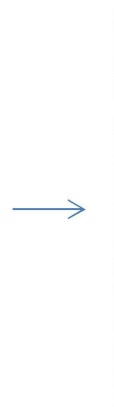

(e)
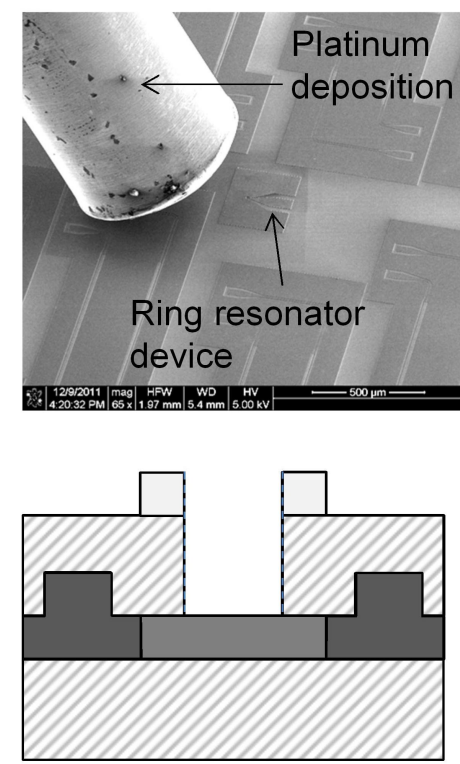

(c)

Refill hole

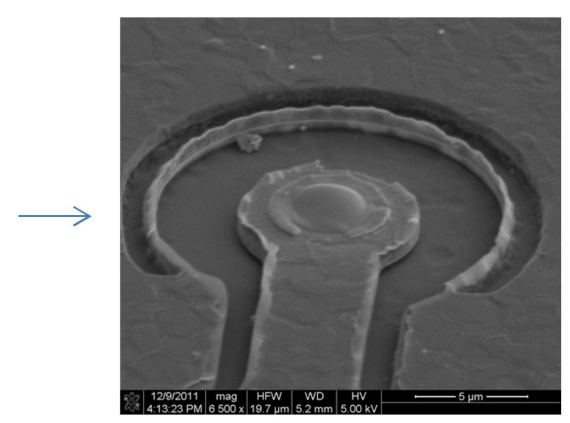

(f)

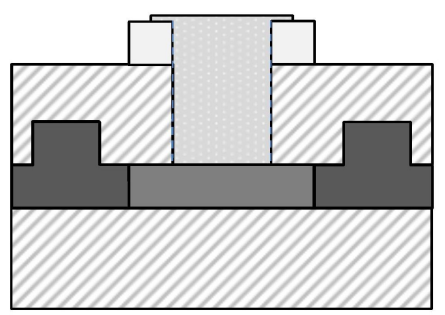

Fig. 2. SEM images showing (a) FIB milled hole in centre electrode, (b) Device post HF dip aligned for platinum deposition, (c) finished device will milled hole refilled with platinum. Also shown are cross-sectional diagrams of the device at different stages in the FIB correction process (note that these do not all match the SEM images above). (d) Device pre-correction, (e) device will hole milled down to the doped slab region and (f) device with milled holed refilled. 
A short dip in hydrofluoric (HF) acid was then used to ensure the top cladding oxide was completely removed from the top of the $p+$ region. The FIB was then used to deposit Platinum, from the precursor methylcyclopentadienyl trimethyl platinum into the milled hole making an electrical contact between the electrode and the doped region. Contact deposition materials available on the FIB were either Platinum or Tungsten. Platinum was chosen as its deposition rate is higher. Scanning electron microscope (SEM) images at different stages of this process are shown in figure 2 . The FIB could prove a very useful tool for this purpose in a research environment especially as photonic circuits become more and more complex.

\section{DEVICE ANALYSIS}

Light from a tunable laser source was passed via an optical fibre to the input surface grating coupler on the optical chip. At the output side of the device light was coupled from the optical chip by another surface grating coupler to an optical fibre terminating at an optical detector. The wavelength of the laser was then scanned and the output power recorded. During the measurements the temperature of the device was stabilised at room temperature. To separate the loss of the device from the coupling losses, a $2 \mu \mathrm{m}$ wide waveguide spanning the length of the chip was also measured for normalisation. Figure 3 shows the normalised spectral response of the ring resonator.

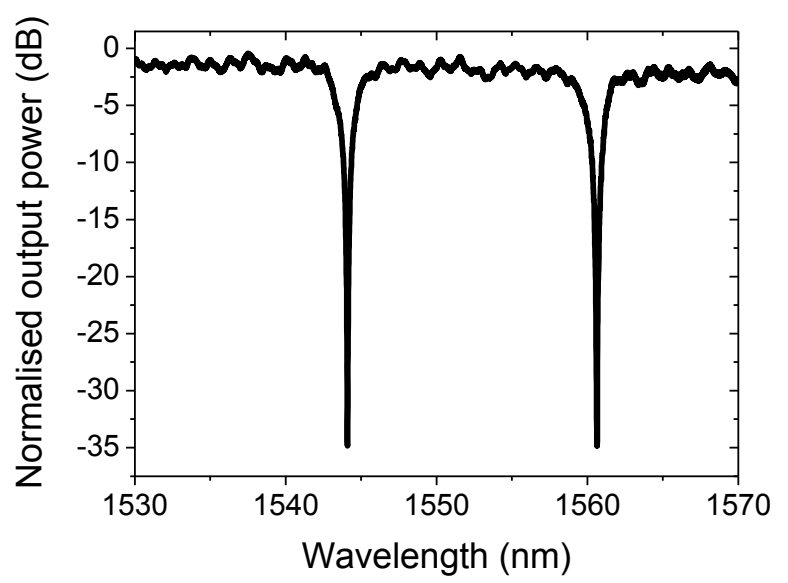

Fig. 3. Normalised passive spectral response of the ring resonator

The off resonance optical loss at the peak of the Fabry-Perot fringes is less than $0.5 \mathrm{~dB}$. Note that this loss also includes the loss due to tapering the waveguide width between $2 \mu \mathrm{m}$ in the input and output sections and $400 \mathrm{~nm}$ in the device section. If multiple rings were cascaded to produce a WDM system for example, only one set of these tapers would be required. The passive extinction ratio is in excess of $30 \mathrm{~dB}$. The full width half maximum (FWHM) is $1.52 \mathrm{~nm}$ corresponding to a $\mathrm{Q}$ of approximately $1 \mathrm{e}^{3}$, and photon lifetime of $0.83 \mathrm{ps}$.

A reverse bias is then applied to the device which causes a shift in the resonance wavelength. This shift has then been used to calculate the modulation efficiency. The modulation efficiency together with that calculated from an MZI structure on the same sample is shown in Figure 4.

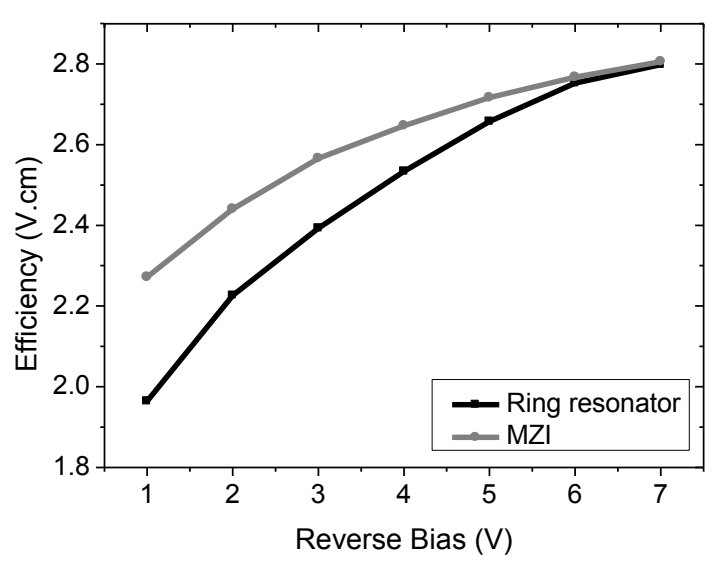

Fig. 4. Change in effective index with reverse bias voltage.

At low voltages (up to $\sim 6 \mathrm{~V}$ ) the ring modulator is more efficient since light is propagating towards the edge of the waveguide and is therefore in greater interaction with the depletion region. As the voltage is increased the depletion region extends more into the waveguide and a greater interaction with the centralised mode of the straight MZI phase modulator occurs. The reverse breakdown voltage of the diode is at approximately $-10 \mathrm{~V}$.

To assess the high speed performance of the device high speed pseudo random bit sequences (PRBS) of length $2^{\wedge} 7-1$ were applied to the electrodes and the optical response monitored on a digital communications analyser (DCA). The PRBS data source was connected to a radio frequency (RF) electrical amplifier. A bias tee was used to apply a DC level of $3 \mathrm{~V}$ to ensure that the device remains in the reverse bias regime during modulation. Due to the mismatch in impedance between

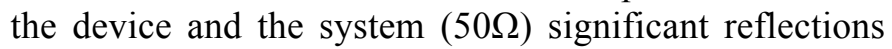
can be observed, as can be seen in the S11 of the device as measured by a vector network analyser (figure 5). These reflections can cause damage to the RF amplifier and distort the PRBS signal. An attenuator is therefore used to suppress these reflections. This results in a reduction of the amplitude of the RF signal passed to the device which has been measured as $1.4 \mathrm{~V}$ peak to peak. However due to the reflection of the RF signal at the ring the voltage across the pn junction could be almost 
twice that measured from the RF amplifier without the modulator connected.

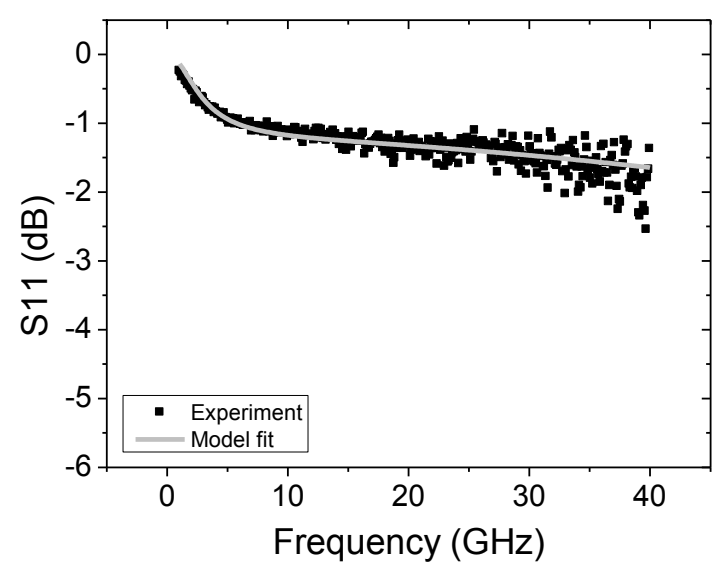

Fig. 5. Device S11 magnitude response

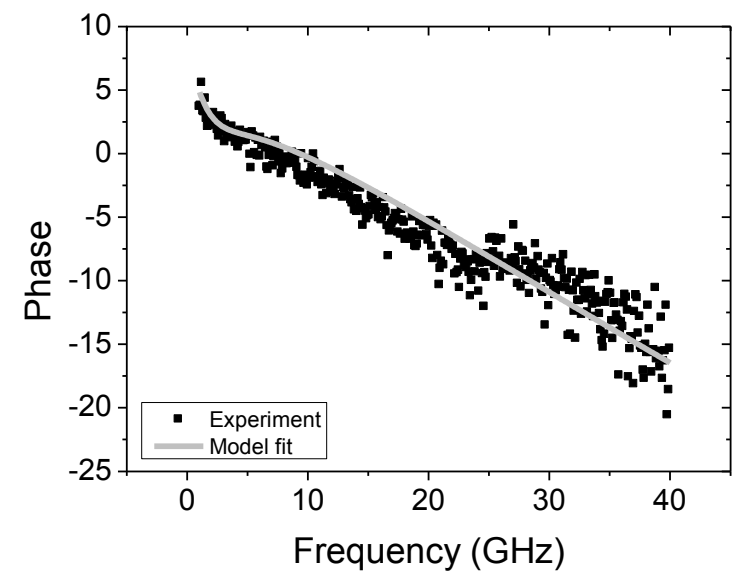

Fig. 6. Device S11 phase response

The optical output of the device was detected by a DCA for the high speed measurements. In order to obtain a sufficient power level to be detected by the DCA the light was passed through an erbium doped fibre amplifier (EFDA). An optical filter was then used to attenuate the noise produced by the EDFA at surrounding wavelengths as much as possible. As significant noise is still present at the DCA the pattern is averaged 128 times. A low Q is required to ensure a short enough photon cavity lifetime to support high speed operation. The photon cavity lifetime in this case is much shorter than required to support $40 \mathrm{Gbit} / \mathrm{s}$ modulation. The negative impact of a low $\mathrm{Q}$ is that the resonance is broader and as a result the change in optical power for a given shift in waveguide is not as large. The extinction ratio produced towards the top of the spectral response is therefore too small to measure the modulation. In order to increase the extinction ratio such as to allow measurement of the eye diagrams in this case the wavelength of the input laser light was set at approximately $1561 \mathrm{~nm}$ during the high speed testing. This results in an additional $10 \mathrm{~dB}$ of loss at the one level. A total insertion loss of $10.5 \mathrm{~dB}$ occurs in this case.

The measurements recorded by the DCA at 20, 25,30 and $40 \mathrm{Gbit} / \mathrm{s}$ can be seen in figure 7 . The extinction ratios measured at the limit of the eye opening at 20,25 and $30 \mathrm{Gbit} / \mathrm{s}$ is $\sim 1.6 \mathrm{~dB}$ whereas at $40 \mathrm{Gbit} / \mathrm{s}$ it is reduced to $\sim 1.1 \mathrm{~dB}$. The reduction in extinction ratio at $40 \mathrm{Gbit} / \mathrm{s}$ can be explained by the imperfect electrical signal feeding the device at $40 \mathrm{Gbit} / \mathrm{s}$ which does not completely reach the 1 and 0 levels during switching as shown in figure 8.

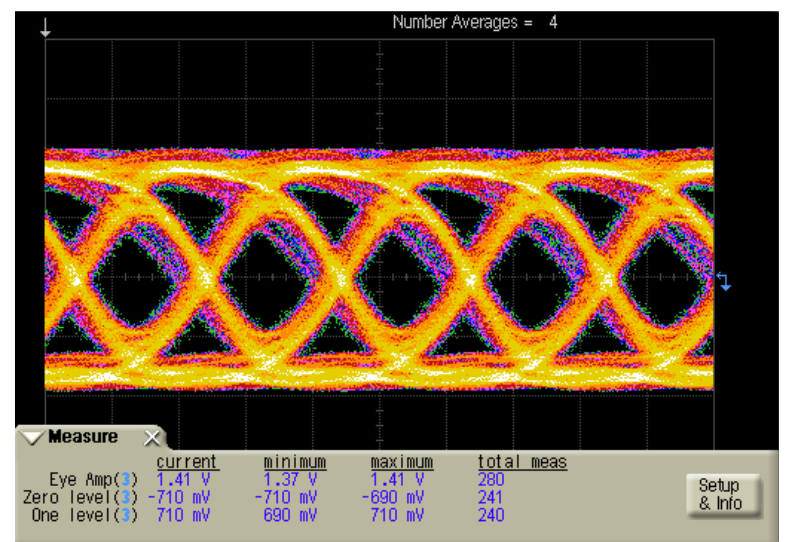

Fig. 8. Electrical drive signal at $40 \mathrm{Gbit} / \mathrm{s}$

Using the technique described in [15], an equivalent circuit model using Advanced Design System (ADS) and the network analyser measurements shown in figure 5 and 6 the junction capacitance of the ring modulator and access resistance has been extracted from the s parameters to be $16 \mathrm{fF}$ and $35 \mathrm{Ohms}$ respectively. Contact resistance at the point of the FIB correction is therefore not expected to have had a significant effect on the bandwidth of the device. The power consumption of the ring modulator can be calculated using equation 1 [9].

$$
P=\frac{C_{J} V^{2}}{4}
$$

Taking the peak to peak drive voltage to be double the $1.4 \mathrm{~V}$ measured results in a worst case power consumption of $32 \mathrm{fJ} / \mathrm{bit}$. 

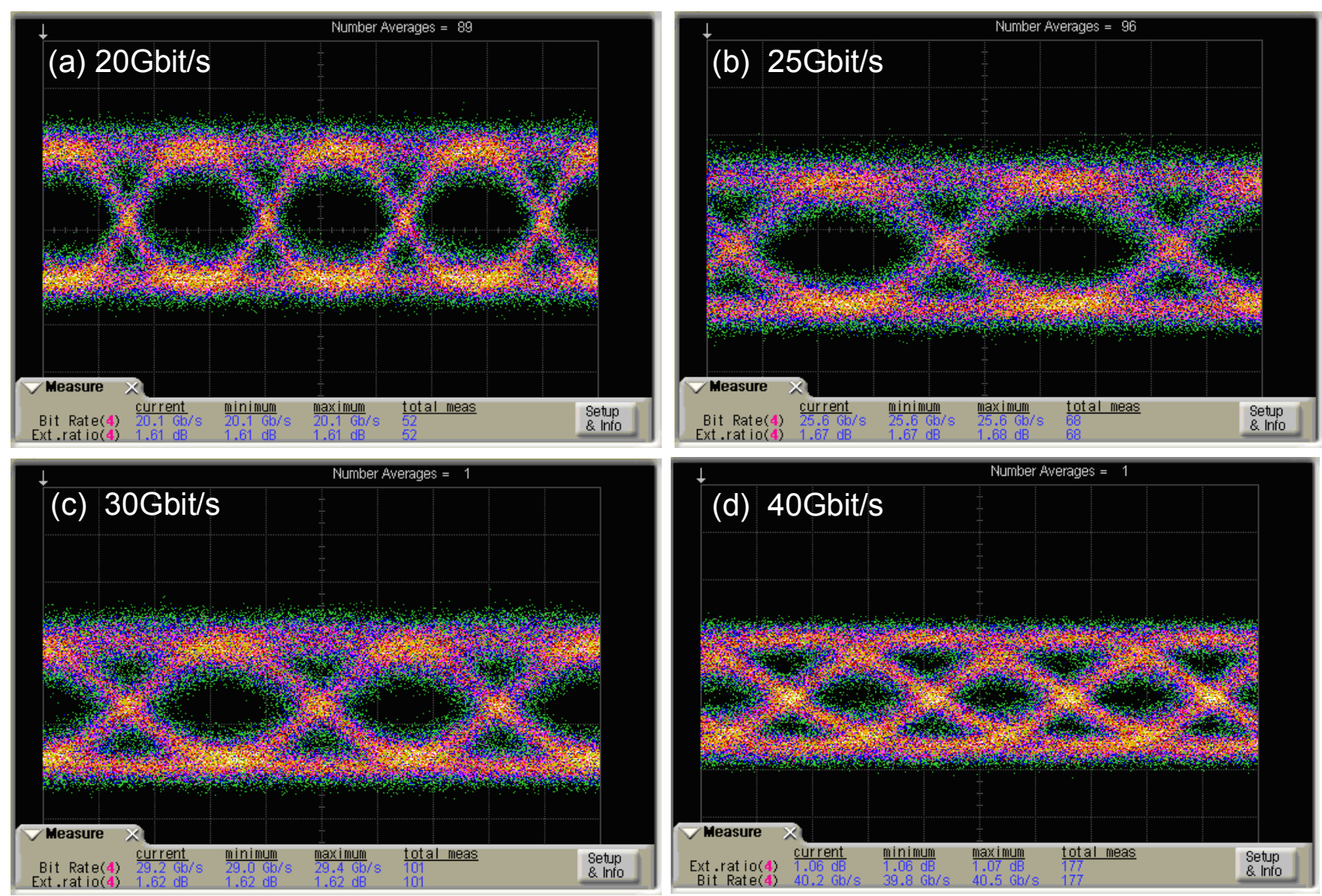

Fig. 7. Optical eye diagram from the ring resonator modulator measured at (a) $20 \mathrm{Gbit} / \mathrm{s}$, (b) $25 \mathrm{Gbit} / \mathrm{s}$, (c) $30 \mathrm{Gbit} / \mathrm{s}$ and (d) 40Gbit/s.

In order to obtain a larger extinction ratio or lower optical loss a larger drive voltage may be used. This approach is however limited practically due to the reverse breakdown of the pn junction and the availability of high voltage drivers. A more practical approach would be to improve the $\mathrm{Q}$ of the ring by using a larger radius. Simulations performed on the passive ring structure which take into account sidewall roughness give a loss figure of approximately $270 \mathrm{~dB} / \mathrm{cm}$ for a $6 \mathrm{um}$ radius. For a $12 \mathrm{um}$ radius this passive loss reduces to $7 \mathrm{~dB} / \mathrm{cm}$. With a 6 um ring radius increased interaction with the highly doped $n$ type region due to the optical mode propagating on the outer edge of the waveguide accounts for approximately a further $30 \mathrm{~dB} / \mathrm{cm}$. This will be reduced if a $12 \mathrm{um}$ ring radius is used. It can be reduced fully if the $\mathrm{n}+$ region is moved $100 \mathrm{~nm}$ further from the waveguide. The increased access resistance due to this can be countered by moving the $\mathrm{p}+$ region $100 \mathrm{~nm}$ closer. Since the $\mathrm{p}+$ region is on the inside of the ring the modal interaction will not be significantly affected. With these modifications a Q of approximately 7500 is calculated. A ring resonance similar to that shown in [22] is therefore expected. With $3 \mathrm{~V}$ applied to our device the resonance shifts by approximately $45 \mathrm{pm}$. If this shift is applied to the device of [22] an extinction ratio of approximately $6 \mathrm{~dB}$ could be obtained with just $2 \mathrm{~dB}$ of additional optical loss at the one level. The Q of 7500 equates to a photon cavity lifetime of approximately $6 \mathrm{ps}$ which does not limit the speed of the device up to 40Gbit/s. It is appreciated that this will result in an increase in power consumption due to both the increased junction length (and therefore capacitance) as well as the slightly increased drive voltage. In this case a power consumption of $72 \mathrm{fJ} / \mathrm{bit}$ results.

\section{SUMMARY}

A compact silicon ring resonator based optical modulator has been demonstrated to operate at high speed. The device is based upon a phase shifter which features self-aligned pn junction formation, which will reduce performance variations in large scale fabrication. A focused ion beam has been used to form one of the contacts due to an error in the mask design, thus demonstrating the value such a tool can play in the development stage of silicon photonic devices and systems. 


\section{ACKNOWLEDGEMENTS}

The research leading to these results has received funding from the European Community's Seventh Framework Programme (FP7/2007-2013) under grant agreement $\mathrm{n}^{\circ} 224312$ HELIOS and from the EPSRC in the UK to support the UK Silicon Photonics project.

\section{REFERENCES}

1. D. J. Thomson, F. Y. Gardes, J-M. Fedeli, S. Zlatanovic, Y. Hu, B. P. -P. Kuo, E. Myslivets, N. Alic, S. Radic, G. Z. Mashanovich and G. T. Reed, "50Gbit/s Silicon Optical Modulator," Photonics technology letters, 24(4), (2011).

2. R. Soref and B. Bennett, "Electrooptical effects in silicon," IEEE Journal of Quantum Electronics, 23(1), (1987)

3. M. Nedeljkovic, R. Soref and G. Z. Mashanovich, "Freecarrier electro-refraction and electro-absorption modulation predictions for silicon over the 1-14um infrared wavelength range," IEEE Photonics Journal, 3(6), (2011)

4. A. Brimont, D. J. Thomson, P. Sanchis, J. Herrera, F. Y. Gardes, J. M. Fedeli, G. T. Reed and J. Martí, "High speed silicon electro-optical modulators enhanced via slow light propagation," Opt. Express, 19(21), 2087620885, (2011).

5. H. C. Nguyen, S. Hashimoto, M. Shinkawa, and T. Baba, "Compact and fast photonic crystal silicon optical modulators," Opt. Express, 20(20), (2012).

6. G. T. Reed, G. Mashanovich, F. Y. Gardes and D. J. Thomson, "Silicon optical modulators," Nature Photonics, 4, 518-526, (2010).

7. D. M. Gill, M. Rasras, K-Y. Tu, Y-K. Chen, A. E. White, S. S. Patel, D. Carothers, A. Pomerene, R. Kamocsai, C. Hill, J. Beattie "Internal bandwidth equalization in a CMOS compatible Siring Modulator," IEEE Photonics Technology Letters, 21(4), 200-2, (2009).

8. F. Y. Gardes, A. Brimont, P. Sanchis, G. Rasigade, D. Marris-Morini, L. O'Faolain, F. Dong, J-M. Fedeli, P. Dumon, L. Vivien, T. F. Krauss, G. T. Reed and J. Martí "High-speed modulation of a compact silicon ring resonator based on a reverse-biased pn diode," Opt. Express, 17(24), 21986-21991, (2009).

9. P. Dong, S. Liao, D. Feng, H. Liang, D. Zheng, R. Shafiiha, C-C. Kung, W. Qian, G. Li, X. Zheng, A. V. Krishnamoorthy and M. Asghari1 "Low Vpp, ultralowenergy, compact, high-speed silicon electro-optic modulator" Opt. Express, 17, 22484-22490, (2009).

10. J-B. You, M. Park, J-W. Park and G. Kim "12.5 Gbps optical modulation of silicon racetrack resonator based on carrier-depletion in asymmetric p-n diode," Opt. Express, 16(22), 18340-18344, (2008).
11. J. C. Rosenberg, W. M. J. Green, S. Assefa, T. Barwicz, M. Yang, S. M. Shank and Y. A. Vlasov "Low=Power 30Gbps Silicon Microring Modulator," Proceedings of CLEO 2011.

12. X. Xiao, X. Li, H. Xu, Y. Hu, K. Xiong, Z. Li, T. Chu, Y. $\mathrm{Yu}$, and J. Yu, "44-Gb/s Silicon Microring Modulators Based on Zigzag PN Junctions," IEEE Photon. Technol. Lett. 24, 1712-1714 (2012).

13. Y. Hu, X. Xiao, H. Xu, X. Li, K. Xiong, Z. Li, T. Chu, Y. $\mathrm{Yu}$, and J. Yu, "High-speed silicon modulator based on cascaded microring resonators," Opt. Express 20, 1507915085 (2012).

14. G. Li, X. Zheng, H. Thacker, J. Yao, Y. Luo, I. Shubin, K. Raj, J. E. Cunningham, and A. V. Krishnamoorthy, "40 $\mathrm{Gb} / \mathrm{s}$ thermally tunable CMOS ring modulator," the 9th Proceedings of IEEE Group IV Photonics, (2012).

15. X, Xiao, H. Xu, X. Li, Y. Hu, K. Xiong, Z. Li, T. Chu, Y. $\mathrm{Yu}$ and J. $\mathrm{Yu}$ "25 Gbit/s silicon microring modulator based on misalignment-tolerant interleaved PN junctions," Opt. Express, 20(3), 2507-2515, (2012).

16. M. Ziebell, D. Marris-Morini, G. Rasigade, P. Crozat, JM. Fedeli, P. Grosse, E. Cassan and L. Vivien "Ten Gbit/s ring resonator silicon modulator based on interdigitated PN junctions," Opt. Express, 19(15), 14690-14695, (2012).

17. P. Dong, R. Shafiiha, S. Liao, H. Liang, N-N. Feng, D. Feng, G. Li, X. Zheng, A. V. Krishnamoorthy and M. Asghari "Wavelength-tunable silicon microring modulator," Opt. Express, 18(11), 10941-10946, (2010).

18. A. M. Gutierrez, A. Brimont, G. Rasigade, M. Ziebell, D. Marris-Morini, J-M. Fedeli, L. Vivien, J. Marti and P. Sanchis "Ring-Assisted Mach-Zehnder Interferometer Silicon Modulator for Enhanced Performance," IEEE Journal of Lightwave Technology, 30(1), 9-14, (2012).

19. W. A. Zortmann, A. L. Lentine, D. C. Trotter, and M. R. Watts. 'Low-voltage differentially-signaled modulators,' Optics Express 19(27), 26017-26026, (2011).

20. G. Li, X. Zheng, J. Lexau, Y. Luo, H. Thacker, P. Dong, S. Liao, D. Feng, D. Zheng, R. Shafiiha, M. Asghari, J. Yao, J. Shi, P. Amberg, N. Pinckney, K. Raj, R. Ho, J. Cunningham and A. V. Krishuamoorthy, "UltralowPower High-Performance Si Photonic Transmitter," Proceedings of OFC 2010.

21. D. J. Thomson, F. Y. Gardes, G. T. Reed, F. Milesi, and J-M Fedeli "High speed silicon optical modulator with self aligned fabrication process," Opt. Express, 18(18), 19064-19069, (2010).

22. G.Li, X. Zheng, J. Yao, H. Thacker, I. Shubin, Y. Luo, K. Raj, J. E. Cunningham, and A. V. Krishnamoorthy " $25 \mathrm{~Gb} / \mathrm{s}$ 1V-driving CMOS ring modulator with integrated thermal tuning," Opt. Express, 19(21), 2043520443, (2011). 